UCRL-JC-132002

\title{
Equation of State of Partially-ionized Plasmas
}

\author{
F. J. Rogers
}

This paper was prepared for submittal to the

Proceedings of International Conference on Strongly Coupled Coulomb Systems Boston, MA

August 3-10, 1997

September 17, 1998

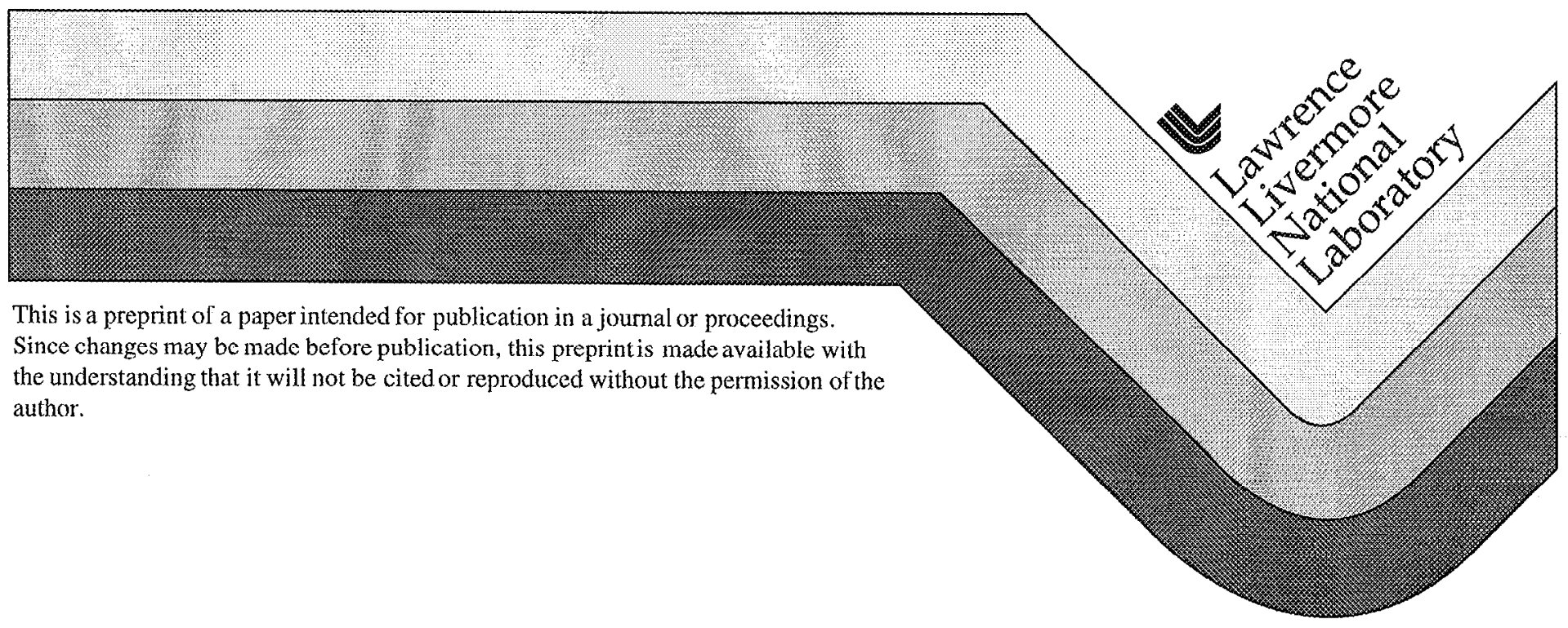




\section{DISCLAIMER}

This document was prepared as an account of work sponsored by an agency of the United States Government. Neither the United States Government nor the University of California nor any of their employees, makes any warranty, express or implied, or assumes any legal liability or responsibility for the accuracy, completeness, or usefulness of any information, apparatus, product, or process disclosed, or represents that its use would not infringe privately owned rights. Reference herein to any specific commercial product, process, or service by trade name, trademark, manufacturer, or otherwise, does not necessarily constitute or imply its endorsement, recommendation, or favoring by the United States Government or the University of California. The views and opinions of authors expressed herein do not necessarily state or reflect those of the United States Government or the University of California, and shall not be used for advertising or product endorsement purposes. 
Forrest J. Rogers

Lawrence Livermore National Laboratory

P. O. Box 808,

Livermore, CA 94550

\section{INTRODUCTION}

The equation of state (EOS) of partially-ionized plasmas has long been of interest in stellar modeling. Stars having masses similar to or greater than the sun are weakly coupled and simple models give results that are adequate for many purposes. However, helioseismology is now able to measure the EOS of the sun, as a function of solar radius, to an accuracy better than $0.1 \%$ [1]. This level of accuracy can not be obtained from simple models and efforts to develop more rigorous theories are in progress.

The Saha equation [2] is the progenitor of methods to treat partially-ionized plasmas. It solves a set of coupled equations that balance ideal gas chemical potentials across chemical reactions occurring in the plasma. In its original form only the isolated particle ground state of each atomic/ionic component was considered. The next simplest approximation adds the classical Debye-Hückel Coulomb term corrected for electron degeneracy. In spite of its simplicity, this is a particularly good model because the deeply bound states are well represented by isolated (atomic) particles and the Debye-Hückel correction contains most of the excited bound state contribution $[3,4]$. Helioseismology supports this theoretical result [5]. The EOS calculations should also include a correction for electron exchange, which is large enough to affect comparisons with helioseismic data [6]. In addition there are small quantum diffraction corrections to the Dehye-Hückel Coulomb term which are not typically included.

For many years it was thought that the next improvement to the Saha equation should be to add excited bound states perturbed by the plasma environment. Since this problem is difficult to treat from fundamental theory, a voluminous literature based on ad hoc approaches was developed. The most commonly used methods to account for environmental effects assume either bound state energies from screened potentials or the confined atom model. This complicates the calculations and it is now known that EOS based on shifted bound state energies are in significantly worse agreement with helioseismology than those using unshifted bound states [7].

Neither EOS theory now commonly used to model stellar plasmas screens bound states. The MHD method [8,9] is a chemical picture approach where the free energy is assumed to be separable into translational, configurational, and Coulomb interaction components. In the chemical picture it is necessary to assert the effect of the plasma on bound states. Based on the null results of Goldsmith, Griem, and Cohen [10], MHD assumes that the bound state energies are unshifted. Instead, plasma effects enter through an occupation probability formalism that separates the Boltzmann sum into effective bound and continuum state parts. The resulting internal partition function is convergent. For neutrals the bound state occupation probability is obtained from a model based on hard sphere interactions between 
atomic cores, while for ions the occupation probability is obtained from the dissolution of states due to electric microfield fluctuations. The MHD Coulomb interactions are given by the classical Debye-Hückel term corrected for electron degenexacy, which effectively limits MIID to weakly coupled plasmas. An ad hoc free energy term is added to guarantee pressure ionization. Electron exchange and diffraction corrections are neglected. The ACTEX method $[3,4,11,12]$, is based on a physical picture approach that carries out a many body activity expansion of the grand canonical partition function (GCPF). A conceptual advantage of the physical picture is that it views the system in terms of its fundamental components; i.e., electrons and nuclei. A natural and important consequence of this approach is that low lying bound states are unscreened. ACTEX includes electron degeneracy and the leading quantum diffraction corrections as well as systematic corrections necessary for strongly-coupled regimes.

Other EOS work aimed mostly at dense astrophysical objects has also recently appeared. In the chemical picture: Saumon, Chabrier, and Van Horn [13] generated EOS tables suitable for modeling low-mass stars and giant planets. An important fcature of their work is the prediction of a plasma phase transition that may affect models of giant planets. Potekhin [14] has generalized the arguments of MHD so that the occupation probability of charged particles is obtained directly without invoking Stark ionization theory. His method does not require an ad hoc free energy term to produce pressure ionization. Stolzmann and Blocker [15] have given a treatment of fully ionized dense stellar matter. They include exchange and charged particle interaction by Padé approximates as well as relativistic effects. In the physical picture: Perrot and Dharma-wardana [16] have given a density-functional theory (DFT) method; Kraeft et al. [17] have developed a thermodynamic Green's function method; Alustuey and Perez [18] have developed a method based on Feynman-Kac path integrals ; Pierleoni et al. [19] have used the restricted path-integral MonteCarlo method to study hydrogen; and Penman, Clérouin, and Zerah [20] have used density functional molecular dynamics to also study hydrogen.

Due to the extreme accuracy required, it is currently not computational feasible to apply the simulation methods to helioseismology. Of the remaining methods the quantum diagrammatic physical picture approaches should give the best comparison with helioseismic data. So far however this has not been the case, primarily due to the difficulty of applying these methods in the region of partial ionization. One problem is that the diagrammatic methods involve a dynamic screened potential that significantly affects the bound states. The ACTEX approach follows a similar line but introduces some simplifications that allow it to treat the region of partial-ionization when the deBroglie wavelength, $\lambda$, is less than the Debye length, $\lambda_{D}$. Consequently, at the corresponding point in the analysis, ACTEX approximates the dynamic screened potential with a static potential having screened energy levels. However, it can be shown that in the region of partial ionization many additional diagrams must be included to treat composite particles on the same basis as fundamental particles. ACTEX accomplishes this by introducing new activity variables that are built from products of the fundamental particle activities and the Boltzmann factors that control the ionization balance between states. The screening corrections to the isolated atomic states introduced through the screened potential are used to define the composite particle activities. Consequently, only isolated atomic energy levels appear in the renormalized expansion. This is where ACTEX goes beyond the more fundamental approaches. A brief description of the ACTEX methodology is given in Section 2. Comparisons to helioseismic and shock data are given in Section 3. 


\section{THE ACTIVITY EXPANSION METHOD FOR PLASMAS (ACTEX)}

Quantum diagrammatic methods are quite complex and it is difficult to get beyond asymptotic limits. However, the main affect of quantum mechanics is to remove the short-ranged electron-ion divergence in the bound state sum. When $\lambda<\lambda_{D}$ it is a good approximation to first develop the classical theory and then replace classical Boltzmann factors with their quantum mechanical analogues. This is the approach taken in ACTEX. At more extreme conditions quantum effects on the distribution of unbound particles become important.

The classical activity expansion of the GCPF of strongly coupled, fully-ionized plasmas involves a many-body analysis of a very large number of both singly connected and multiply connected diagrams. In contrast, only the multiply connected diagrams contribute to a density expansion of the canonical partition function. Abe [21] showed how to carry out an all-orders expansion in the density. The leading terms in the resulting convergent multi-component expression for the non-ideal Helmholtz free energy are

$$
\frac{F-F_{0}}{V k T} \equiv S=S_{R}+\sum_{i j} S_{i j}+\sum_{i j k} S_{i j k} \cdots
$$

where the indices $i, j, k$ etc. range over all components,

$$
S_{R}=\frac{1}{12 \pi \lambda_{D}^{3}}
$$

is the ring diagram sum,

$$
S_{i j}=-n_{i} n_{j}\left[B_{i j}\left(T, \lambda_{D}\right)+2 \pi \int_{0}^{\infty} r^{2} d r\left(\beta u_{i j}-\frac{\beta u_{i j}}{2}\right)\right]
$$

is closely related to the second virial coefficient, $\mathrm{B}_{\mathrm{ij}}$, of the static screened potential

$$
u_{i j}=\frac{Z_{i} Z_{j} e^{2} e^{-r / \lambda_{D}}}{r},
$$

the n's number fractions, the Z's are particle charges and,

$$
\lambda_{D}=\sqrt{\frac{k T}{4 \pi e^{2} \sum_{j} n_{j} Z_{j}^{2}}},
$$

is the Debye length. The $S_{i j k}$, and higher order terms systematically replace the divergent Coulomb virial coefficients with the virial coefficients for the Debye-Hückel potential (equation.(4)). The terms through order $n^{2}$, given by equations. (2-5), show that there are some differences in detail. For example, there appears a term of order $\mathrm{n}^{3 / 2}$, i.e. the Debye-Hückel Coulomb interaction term, coming from the ring diagrams, while terms of order $\beta u_{i j} j$ and $\left(\beta u_{i j}\right)^{2}$ are missing from the screened second virial coefficient.

Equation (1) is a complete many-body solution for classical multi-component plasmas. Consequently, even though it is an expansion away from weak coupling, it 
recovers the Monte-Carlo strong coupling result [12]. It is well known that the grand canonical ensemble $(\mathrm{GCE})$ is the appropriate choice for partially-ionized plasmas. It would be difficult to obtain a classical GCE result similar to the Abe result for the canonical ensemble. The corresponding quantum-mechanical expression, would be even more difficult to develop. The ACTEX method sidesteps both of these problems. The classical GCE result for fully ionized multi-component plasmas is obtained from a procedure that inverts equation (1) in terms of $S\left(T, n_{(i)}\right)$ to obtain an expression for $\mathrm{P} / \mathrm{kT}$ as a functional of $S\left(T, z_{\{i\}}\right)$ The result for a two component plasma of electrons, $e$, and nuclei, $\alpha$, of charge $Z$ is

$$
\frac{P}{k T}=z_{e}+z_{\alpha}+S+\sum_{m=2}^{\infty} \frac{z_{e}}{m !}\left(\frac{\partial}{\partial z_{e}} z_{e}\right)^{m-2}\left(\frac{\partial S}{\partial z_{e}}\right)^{m}+\sum_{m=2}^{\infty} \frac{z_{\alpha}}{m !}\left(\frac{\partial}{\partial z_{\alpha}} z_{\alpha}\right)^{m-2}\left(\frac{\partial S}{\partial z_{\alpha}}\right)^{m}+\cdots
$$

where, the ellipsis indicates that cross terms not necessary for the present discussion have been left out and the

$$
z_{i}=\left(2 s_{i}+1\right) \lambda_{i}^{3} e^{\mu_{i} / k T}, i=\{e, \alpha\}
$$

are the activities.

As it stands, equation (6) is not very useful, since it involves operations on the function $S$ which is a sum over virial coefficients, whereas, a properly constructed activity expansion of the GCPF should involve cluster coefficients. However, it is a complete result that can be reorganized in terms of cluster coefficients of the screened potential. For a two component plasma of electrons, e, and heavy ions of type, $\alpha$, the reorganized expression through terms of order $z^{5 / 2}$ is [12]:

$$
\begin{aligned}
\frac{P}{k T}= & z_{e}+z_{\alpha}+S_{R}\left(U^{*}\right)+\frac{z_{e}}{2 !}\left(\frac{\partial S_{R}}{\partial z_{e}}\right)^{2}+\frac{z_{\alpha}}{2 !}\left(\frac{\partial S_{R}}{\partial z_{\alpha}}\right)^{2}+\cdots+z_{e}^{2} c_{e e}+2 z_{e} z_{\alpha} c_{e \alpha}+z_{\alpha}^{2} c_{\alpha \alpha} \\
& +z_{e} \frac{\partial S_{R}\left(U^{*}\right)}{\partial z_{e}} \frac{\partial}{\partial z_{e}}\left(z_{e}^{2} c_{e e}+2 z_{e} z_{\alpha} c_{e \alpha}+z_{\alpha \alpha}^{2} c_{\alpha \alpha}\right) \\
& +z_{\alpha} \frac{\partial S_{R}\left(U^{*}\right)}{\partial z_{\alpha}} \frac{\partial}{\partial z_{\alpha}}\left(z_{e}^{2} c_{e e}+2 z_{e} z_{\alpha} c_{e \alpha}+z_{\alpha}^{2} c_{\alpha \alpha}\right)
\end{aligned}
$$

subject to the conditions,

$$
n_{e}=z_{e} \frac{\partial}{\partial z_{e}}\left(\frac{P}{k T}\right) \quad, \quad n_{\alpha}=z_{\alpha} \frac{\partial}{\partial z_{\alpha}}\left(\frac{P}{k T}\right) .
$$

In equation (8),

$$
S_{R}\left(U^{*}\right)=\frac{1}{12 \pi \lambda_{A}^{3}\left(U^{*}\right)}
$$

is the ring diagram sum, similar to equation (2),

$$
\lambda_{A}\left(U^{*}\right)=\sqrt{\frac{k T}{4 \pi e^{2} U^{*}}}
$$

is the screening length in the GCE, and 


$$
U^{*}=z_{e}+Z^{2} z_{\alpha}
$$

is defined for later convenience (the asterisk indicates that $U^{*}$ is not in final form), the

$$
c_{i j}=b_{i j}-b_{i j}^{1}-b_{i j}^{2}
$$

will be referred to as Coulomb cluster coefficients, the $b_{i j}$ are screened second cluster coefficients and $\mathrm{i}, \mathrm{j}=\{\mathrm{e}, \alpha\}$. For two body terms $b_{i j}=-B_{i j}$. The ellipsis in equation (8) indicates that terms of order $z^{5 / 2}$ not needed in the present discussion are not included.

The second deficiency of equation (8), i.e., the Coulomb short range divergence, can now be removed by replacing classical Boltzmann factors with their quantum analogs. For example, the screened classical second clustex coefficient for the e- $\alpha$ interaction is replaced by

$$
b_{e \alpha}=4 \pi \pi_{e \alpha}^{3} \operatorname{Tr}\left(e^{-\beta H_{2}}-e^{-\beta H_{0}}\right)
$$

where the trace is over the states of the potential

$$
u_{e \alpha}=-Z e^{2} \frac{e^{-r / \lambda_{A}}}{r}, \text { and } \quad \lambda_{e \alpha}=\sqrt{\frac{\hbar^{2}}{2 \mu_{e \alpha} k T}}
$$

is the thermal deBroglie wavelength. Beth and Uhlenbeck [22] have shown that equation (14) can be expressed in the convenient form

$$
b_{e \alpha}=4 \pi^{3 / 2}\left[\lambda_{e \alpha}^{3} \sum_{n l}(2 l+1) e^{-\beta E_{n l}}+\frac{1}{\pi} \sum_{l}(2 l+1) \int_{0}^{\infty} \frac{d \delta_{l}}{d p} e^{-\beta p^{2} / 2 \mu_{e \alpha}}\right]
$$

where the $E_{n l}$ are bound states and the $\delta_{l}$ are phase shifts. Only the phase shift term contributes to the e-e cluster coefficient, while the $\alpha-\alpha$ second cluster coefficient can be calculated from the classical expression:

$$
b_{\alpha \alpha}=2 \pi \int_{0}^{\infty}\left[e^{-\beta u\left(r, \lambda_{A}\right)}-1\right] r^{2} d r
$$

At low temperature the ground state Boltzmann factor in equation (15) completely dominates the sum, indicating that the equilibrium state is largely composed of hydrogenic bound pairs. It is easy to show that in this situation the product $2 z_{e} z_{\alpha} c_{e \alpha}^{1 s}$ in equation (13) is the activity of hydrogenic pairs [12,23]. It follows that in partially-ionized plasmas every occurrence of the product $2 z_{e} z_{\alpha} c_{e x}$ should be decomposed into linear and quadratic parts. In general, all occurrences of terms involving electron-ion Coulomb cluster coefficients for $\mathrm{N}$ particles should be decomposed into $\mathrm{N}$ terms of order 1 to $\mathrm{N}$ in the activity. In the following we refer to the 1 s hydrogenic activity as $z_{e \alpha}^{*}$, dropping the superscript for simplicity, i.e., 


$$
z_{e \alpha}^{*}=8 \pi^{3 / 2} \lambda_{e \alpha}^{3}(2 l+1) e^{-\beta E_{1 s}\left(U^{*}\right)} z_{e} z_{\alpha}
$$

In practice this is not the definition of $z_{e x}^{*}$ actually used. Compensation [12, 24] between the bound and scattering state parts of equation (15) replace $e^{\beta E_{1 s}}$

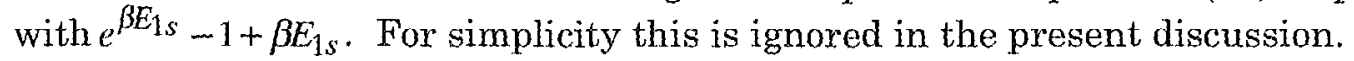

The next step in the ACTEX procedure is to reorganize equation (8) to account for the formation of composite particles as the temperature is lowered. To introduce composite particles on an equal footing with fundamental particles, it is necessary to find terms such that $z_{e \alpha}^{*}$ appears as a new variable similar to fundamental particles. To lowest order the reorganized expression for the pressure is $P / k T=z_{e}+z_{\alpha}+z_{e x}^{*}$, where the new activity for hydrogenic ions becomes from the term $2 z_{e} z_{\alpha} c_{e \alpha}$. At the ring sum level (equation (10)) things are already much more complicated. In order to introduce the screening of hydrogenic charges into the Debye length, i.e., replace $U^{*}$ with $U^{*}+(Z-1)^{2} z_{e \alpha}^{*}$ it is necessary to find all the terms in the transformation

$$
S_{R}\left(U^{*}\right) \rightarrow S_{R}\left(U^{*}+(Z-1)^{2} z_{e \alpha}^{*}\right)
$$

To start this process note that (see equation (8)), ignoring the term involving $\partial c_{e \alpha} / \partial z_{e}$,

$$
z_{e} \frac{\partial S_{R}\left(U^{*}\right)}{\partial z_{e}} \frac{\partial\left(2 z_{e} z_{\alpha} c_{e \alpha}\right)}{\partial z_{e}} \equiv 2 z_{e} z_{\alpha} c_{e \alpha} \frac{\partial S_{R}\left(U^{*}\right)}{\partial z_{e}}=z_{e \alpha}^{*} \frac{\partial S_{R}\left(U^{*}\right)}{\partial z_{e}}
$$

which is $3 / 2$ order in $z_{e \chi}^{*}$, similar to equation (10). Collecting all terms of order $z_{e \alpha}^{* 3 / 2}$ in equation (8) gives

$$
S_{R}\left(U^{*}\right)+z_{e \alpha}^{*} \frac{\partial S_{R}\left(U^{*}\right)}{\partial z_{e}}+z_{e \alpha}^{*} \frac{\partial S_{R}\left(U^{*}\right)}{\partial z_{\alpha}}=S_{R}\left(U^{*}\right)\left[1+\left(Z^{2}+1\right) z_{e \alpha}^{*} \frac{\partial S_{R}}{\partial U^{*}}\right]
$$

This is close to what is needed in 1st order to make the transformation (18), except a cross term proportional to $\mathrm{Z}$ is absent. A term $\propto \mathrm{Z}$ would have to come from e- $\alpha$ interactions and in fact can be found in $z_{e \alpha}^{*}$.

Consider a perturbation expansion of $E_{n l}\left(\lambda_{A}\right) / k T$ :

$$
\frac{E_{n l}\left(\lambda_{A}\right)}{k T}=\frac{E_{n l}^{0}}{k T}+\frac{Z e^{2}}{k T \lambda_{A}}-\frac{a_{0} e^{2}}{4 k T \lambda_{A}^{2}}\left[3 n^{2}-l(l+1)\right]+\cdots
$$

The linear shift is the same for all states and can be written in the suggestive form

$$
\frac{Z e^{2}}{k T \lambda_{A}}=2 Z \frac{\partial S_{R}\left(U^{*}\right)}{\partial U^{*}}
$$

Using this relation we can rewrite equation (21) in terms of the shifted Debye energy levels [25] according to

$$
\frac{E_{n l}}{k T}=\frac{E_{n l}^{s}}{k T}+2 Z \frac{\partial S_{R}\left(U^{*}\right)}{\partial U^{*}}
$$


Limiting the discussion to just the ground state activity and using (23) in (17) gives

$$
z_{e \alpha}^{*}=8 \pi^{3 / 2} \lambda_{e \alpha}^{3}(2 l+1) e^{-\beta E_{1 s^{s}}^{s}} e^{-2 Z \frac{\partial S_{R}\left(U^{*}\right)}{\partial U^{*}}} z_{e} z_{\alpha} .
$$

equation. (24) can be used to define an activity for hydrogenic ions in terms of the shifted Debye energy levels according to

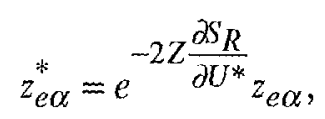

where,

$$
z_{e \alpha}=8 \pi^{3 / 2} \lambda_{e \alpha}^{3}(2 l+1) e^{-\beta E_{1 s}^{s}} z_{e} z_{\alpha}
$$

Next, expanding the exponential term involving $\partial S / \partial U$ gives

$$
z_{e \alpha}^{*}=\left(1-2 Z \frac{\partial S_{R}}{\partial U^{*}}+\cdots\right) z_{e \alpha}\left(U^{*}\right)
$$

The second term in equation (27) is just what is needed to give the factor $(\mathrm{Z}-1)^{2}$ in equation (20). Repeating the process for higher order terms in the expansion of $S_{R}(U)$ eventually gives the result

$$
S_{R}\left(U^{*}\right)+(Z-1)^{2} z_{e \alpha} \frac{\partial S_{R}}{\partial U^{*}}+\cdots=S_{R}(U)
$$

where

$$
U=z_{e}+Z^{2} z_{\alpha}+(Z-1)^{2} z_{e \alpha}
$$

Continuing in this way for higher order terms in the activity gives the transformation $\frac{P}{k T}\left(z_{e}, z_{\alpha}\right) \rightarrow \frac{P}{k T}\left(z_{e}, z_{\alpha}, z_{e \alpha}\right)$, plus extra terms. However, these extra terms obey simple relations. For example, the sum of all terms of order $z_{e \alpha}$ have the important property:

$$
z_{e \alpha}(U)-U \frac{\partial z_{e \alpha}}{\partial U}+\frac{U^{2}}{2} \frac{\partial^{2} z_{e \alpha}}{\partial U^{2}}+\cdots=z_{e \alpha}(U-U)=z_{e \alpha}(0),
$$

Higher order terms display similar properties. The introduction of an augmented set of activities to account for the shifting ionization balance as the temperature and density change, results in an expansion in terms of $E_{1 s}^{0}$, i. e. $E_{1 s}^{s} \rightarrow E_{1 s l}^{0}$. The result is similar for excited states except near the plasma continuum [3].

The discussion so far has been limited to Boltzmann statistics and weak coupling. This has laid out the essential steps of the method and shows how the addition of diagrams in the region of partial-ionization, which are not included in more fundamental calculations [12], leads to an important result regarding bound states. The generalization of the ACTEX method to include the affect of electron degeneracy is accomplished using the method of Cooper and DeWitt [26] The effect of exchange is also added. Quantum diffraction corrections for low order texms are obtained from DeWitt [27]. A method for adding strong ion coupling is given in [12]. 


\section{COMPARISON WITH SEISMIC AND EXPERIMENTAL DATA}

Until recently it was not possible to validate theories of the EOS of partially-ionized plasmas by other means. That situation has changed dramatically. Now there are not only large projects aimed at observing the seismology of the sun to obtain very accurate EOS data, but also laser techniques that measure the shock Hugoniot. There have becn a number of interesting comparisons between these observational and experimental projects that can be mentioned only briefly (see the papers by Däppen, Kress et al., and Militzer et al. elsewhere this Volume).

A number of comparisons of the MHD and ACTEX equations of state have been made to helioseismic inversions [28-30]. In general both equations of state are in good agreement with the data, but these studies have found that the ACTEX EOS yields better consistency. Basu and Christensen-Dalsgaard [31] have recently used the newly available LOW L data [32] to compare the difference in $\Gamma_{1}=(\partial \ln P / \partial \ln \rho)_{s}$ between the sun and theories. In this case neither of the calculations stands out as a significantly better match with the data, although ACTEX matches slightly better overall. Analysis of higher-degree data will improve the quality of the inversions near the surface, where the greatest theoretical discrepancies occur. Models using the ACTEX EOS reduced calculated globular cluster ages by about a billion years, in better agreement with cosmology [33]

Gas gun [34] and laser shock measurements [35] of the deuterium Hugoniot present a challenge to theory. Starting from a liquid state with a density $\rho_{0}=0.171$ g/cc these experiments have reached pressures up to 2.1 Mbar (see Fig.(1)), The Hugoniot data displays a large maximum in the compression, a value of six at a pressure of 1.5 Mbar, although for most materials the maximum compression along the Hugoniot generally does not exceed 4.5. The unusually large maximum compression ratio in deuterium is due to the large internal energy of the diatomic initial state. The shock temperature was not measured, but calculations give a value around $3.5 \mathrm{eV}$ at $2 \mathrm{Mbar}$. Under these conditions deuterium is mostly dissociated and about $15 \%$ ionized. There are large discrepancies between the theories and between the theories and experiment. Tight-binding MD calculations [36] give only a slight maximum near $\rho / \rho_{0}=4$, while quantum $\mathrm{MC}$ calculations [9] give a maximum compression ratio around 5 at pressure of $0.7 \mathrm{Mbar}$. That there are such large discrepancies between the simulations as well as between the simulations and experiment is a puzzle. The SESAME model [37] gives results similar to MD. The Thomas-Fermi-based QEOS model [38] gives a bump at about the same pressure as experiment, but at substantially lower density. Linear mixing model [39] calculations agree well with the experimental data, except at the highest pressures. The ACTEX calculations are in good agreement with the highest pressure data, but they do not include molecules and so do not extend to the low pressure regime.

Comparisons of ACTEX with high density gas gun and nuclear shock data for $\mathrm{Be}, \mathrm{Al}, \mathrm{CH}, \mathrm{H}_{2} \mathrm{O}$, and $\mathrm{SiO}_{2}$ were recently used to validate [40] the method for $\mathrm{r}$ up to 9 .

\section{DISCUSSION}

There are plans to improve the range and the quality of EOS data obtained from helio- and astro-seismic observations as well as laser shock experiments. This will present an increasing challenge to ongoing efforts to model the seismic data from physical picture approaches. The existing discrepancies between MD and MC calculations and experiment are already causing considerable effort to understand the 
reasons and should lead to improvements in these basic approaches. In the next few years it can be expected that these new experimental and theoretical efforts will expand our understanding of partially-ionized regime.

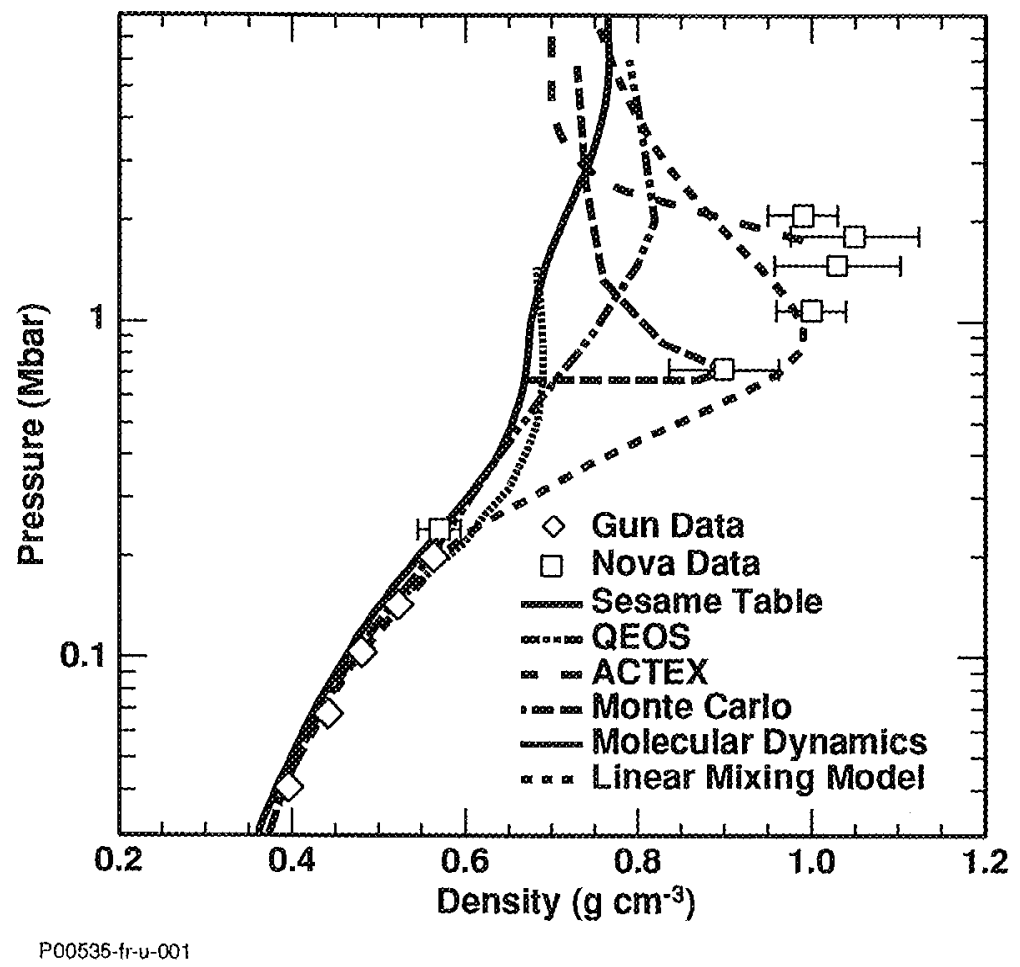

Fig.1 Comparison of theory and experiment for the deuterium Hugoniot.

\section{ACKNOWLEDGMENTS}

I wish to thank Burkhard Militzer for providing QMC $\mathrm{D}_{2}$ Hugoniot data and Robert Cauble for providing Figure 1. This work was performed under the auspices of the $U$. S. Department of Energy by the Lawrence Livermore National Laboratory under Contract W-7405-Eng-48

\section{REFERENCES}

1. J. Christensen-Dalsgaard et al., Science, 272:1286 (1996)

2. M. Saha, Phil. Mag. 40: 472 (1920)

3. F. J. Rogers, ApJ., 310: 723 (1986)

4. F. J. Rogers, "Equation of State In Astrophysics", eds G. Chabrier and E. Schatzman, Cambridge Univ. Press, Cambridge, (1994)

5. J. Christensen-Dalsgaard and D. Däppen, A\&A. Rev. 4: 267 (1992)

6. J. A. Guzik and F. J. Swenson, , ApJ (in press)

7. S. Arndt., W. Däppen, and A. Nayfonov, ApJ, (in press)

8. D. G. Hummer and D. Mihalas,, ApJ., 331: 794 (1988)

9. D. Mihalas, W.. Däppen, and D. G. Hummer ApJ, 331:815 (1988)

10. S Goldsmith, H. E. Griem., and L. Cohen, Phys. Rev., A 30: 2775 (1984)

11. F. J. Rogers, F. J. Swenson, and C. A. Iglesias, ApJ., $456: 902$ (1996)

12. F. J. Rogers, Phys. Rev. A24: 1531 (1981).

13. D. Saumon, G. Chabrier, and H. M. Van Horn. ApJS, 99: 713 (1995)

15 W. Stolzmann. and T. Blocker, A \&A, 314: 365 (1996) 
16. F. Perrot and . W. C. Dharma-wardana, Phys. Rev E. 52:5352 (1995)

17. D. Kremp, W. D. Kraeft, M. Schlanges, Contrib. Plasma Phys., 33:567 (1993);

W. D. Kraeft, et al., Zeitschr. f. Phys. Chem. (in Press)

18 A. Alustuey, and A. Perez, Phys. Rev. E, 53,5714 (1996).

19. W. R. Magro, D. M. Ceperley, C. Pierleoni, and B. Bernu, Phys. Rev. Lett, $76: 1240$ (1996); C. Pierleoni, et al. Phys. Rev. Lett, 73:2145 (1994)

20. J. I. Penman, J. G. Clérouin, and P. G. Zerah, Phys Rev. E., 51:R5224 (1995)

21. R. Abe, Prog. Theor. Phys., 22:213 (1959)

22. E. Beth and G. E. Uhlenbeck, Physica, 3:915 (1937)

23. F. J. Rogers and H. E. DeWitt, Phys. Rev. A, 8:1061 (1973)

24 C. Pisano and H. J. McKeller, Phys. Rev. A, 40:6597 (1989)

25 J. L. Jackson and L. S. Klein, Phys. Rev., 177:352 (1969)

26. M. S. Cooper and H. E. DeWitt, Phys. Rev. A, 8:1910 (1973)

27. H. E. DeWitt, J. Math. Phys. 7:161 (1966)

28. W. A. Driembowski., A. A. Pamyatnykh, and R. Sienkiewicz, Acta Astr. 14: 5 (1992)

29. J. Christensen-Dalsgaard et al., Science, 272: 1286 (1996)

30. J. N. Bahcall, M. H. Pinsonneault., S. Basu, L. Christensen-Dalsgaard, Phys. Rev. Lett., 78:171 (1997)

31. S. Basu. and J. Christensen-Dalsgaard, J. 1997, ApJ 322, L5

32. Tomczyk, S., Card, K., Elmore, D., Hull, H., and Caccini, A. 1995, Solar Phys, 159,1

33. Salaris, M., Degl'Innocenti, S., and Weiss, A. 1997, A \& A, 479, 665

34. W. J. Nellis et al., J. Chem. Phys., 79:1480 (1983)

35. DaSilva, L. B, et al. 1992, Phys. Rev. Lett, 69, 438

36. T. J. Lenosky, J. L. Kress, and L. A. Collins, Phys. Rev. B, 56:5164 (1997)

37. G. I. Kerley, "Molecular-Based Study of Fluids", eds. J. M. Haile and D. A . Mansoori, American Chemical Society, Washington, D. C., (1983)

38 R. M. More, K. H. Warren, D. A. Young, G. B. Zimerman, Phys. Fluids, 31:3059 (1988)

39. M. Ross, Phys. Rev. E, (submitted)

40. F. J. Rogers and D. A. Young, Phys Rev. E, (in press) 